\title{
Characteristics of species composition, biochemical and pathogenic nature of the microbiota of mammary gland and the reproductive tract in dairy cows
}

\author{
O.V. Sokolova ${ }^{*}$, N.A. Bezborodova, Y.Y. Lysova, and E.V. Pechura \\ Ural Federal Agrarian Research Center of the Ural Branch of Russian Academy of Sciences
}

\begin{abstract}
This research is dedicated to a relevant topic in veterinary medicine, which is connected with studying of specific features in systems that are responsible for productive and reproductive functioning in cattle. The reported study was funded by RFBR and Sverdlovsk region, project number 20-416-660004 «Molecular genetic and phenotypic characteristics of the microbiota in the reproductive system of cattle». Samples of secretory product of the mammary gland and cervical swabs of 81 cows and 5 dairy farms of Sverdlovsk region (Russia) were analyzed. A number of pathogenic and opportunistic microorganisms (S.aureus, S.lugdunensis, Streptococcus spp., Enterobacter spp., Enterococcus faecium, Enterococcus faecalis, Escherichia coli, Proteus vulgaris, Bacillus spp.)and mold fungi (Mucor spp., Penicillum spp., Aspergillus spp.) were isolated as a result of microbiological testing of the biological samples. The microbiota structure of the reproductive tract and the lacteal gland is demonstrated in physiologically normal state and during the inflammatory process. The phenotypic characteristic of isolated microorganisms, as well as evaluation of biochemical parameters and pathogenicity were indicated.
\end{abstract}

\section{Introduction}

Microbiota of the reproductive tract and the mammary gland has a significant value in mammal's microbiome due to its ability to influence on the fetus by translocation bacteria and their particles through placental barrier and taking a part in colonization of newborn's intestines. Furthermore, the composition of row milk microbiota, which is associated with microbiogeocenosis of animal's mammary gland is a main factor in terms of both spoiladge and food safety, which effects on quality of the milk intended for direct consumption and subsequent processing [1-3].

Earlier studies suggested that the mammary gland is a sterile organ and there were no microorganisms in tissue. However, lately, scientists at Western University of Canada had proven that these tissues have their own intramammary microbiota. Nevertheless, the scope

\footnotetext{
*Corresponding author: nauka_sokolova@mail.ru
} 
and origin of microbial colonization are still under discussion [4]. According to updated scientific literature, the composition of milk microbiota is defined by contacting with two main sources. Direct contact is associated with microbial ecosystems of teat's canal and skin in dairy cows. Indirect contact is related to various elements of the environment, such as bedding, faeces, nutrition, potable water, air, milking machine and other equipment [5]. Several studies support the hypothesis that bacteria appear in milk not only as a result of external colonization, since bacterial isolates that were presented in the mammary gland had been discovered to be genotypically different from the same species found on the skin of the same host [6].

In foreign and native literature there are numerous data on the reproductive tract microbiocenosis in cows [7]. The understanding of the uterus microbiota of healthy dairy cows and the role of microbiota dysbiosis in the development of metritis has been expanded [8-10]. The role of the uterine microbiota in the cyclicity of the ovaries has been deduced, it has an influence on uterine involution in the postpartum period [11]. There is evidence of the cervical microbiota composition that was obtained by sequencing cows in different physiological phases, including the postpartum period, the period before fertilization and the period of gestation, as well as in cows with metritis. The results revealed a complex profile with a wide range of dissimilarities in the composition of cervical bacteria, requiring further study of the relationship between various factors [12]. In recent literature, findings have emerged about interaction between bacteria and the immune system of the intestines, uterus and the mammary gland in cattle [13]. However, the studies did not take into account the peculiarities of a particular region. Meanwhile, there is evidence that regional geographical and ecological features have an impact on the structure of the organism microbiome and the milk microbiota in cattle [14]. The Sverdlovsk region is a representative of a complex ecological situation due to the heavy industry and transport network development $[2,15]$. A comprehensive study of the mammary gland and the reproductive tract microbiota structure in cows, including the study of the phenotypic properties of microorganisms, will expand the perception of the pathogenesis of diseases and develop an ecosystem-based approach to their treatment and prevention in the region.

The goal of the research - to define composition, biochemical and pathogenic properties of the mammary gland and the reproductive tract microbiota in dairy cows in physiologically normal state and during the pathology of inflammatory nature.

\section{Materials and Methods}

This analysis work was realized in Ural Federal Agrarian Research Center of the Ural Branch of Russian Academy of Sciences in the Sverdlovsk region in 2020. The reported study was funded by RFBR and Sverdlovsk region, project number 20-416-660004 «Molecular genetic and phenotypic characteristics of the microbiota in the reproductive system of cattle».

\subsection{Formation of experimental groups of animals}

Formation of experimental groups of animals was conducted from 5 dairy farms in the Sverdlovsk region, in each of that groups lactating Russian Black Pied cattle breed were selected $(\mathrm{n}=81)$ :

Group 1 - animals without signs of inflammation of the mammary gland and the reproductive tract $(\mathrm{n}=22)$;

Group 2 - animals with signs of inflammation of the mammary gland, but with no signs of the reproductive tract inflammation $(n=24)$; 
Group 3 - animals with signs of inflammation of the reproductive tract, but with no signs of the mammary gland inflammation $(n=18)$;

Group 4 - animals with inflammation of the reproductive tract and the mammary gland $(n=17)$.

Diagnostics of the animal's mammary gland condition was exploit on the basis of clinical research methods (examination, palpation), organoleptic estimation of the mammary gland secretion and determination of the level of somatic cells located in milk. For this purpose, an express test was carried out using the "Kenotest" diagnostic system (CID Lines, Belgium). Diagnostics of inflammatory diseases of the reproductive organs in cows was performed by vaginal and transrectal examination. Moreover, the topography of reproductive organs, contractile function of uterus and characteristics of pathological exudate were investigated.

\subsection{Selection of biological materials samples for the research}

Samples of biological material (the mammary gland secretion, cervical swabs) were selected from each cow of all experimental groups.

After pre-milking teat preparation procedures milk from all quarters of the mammary gland was collected into sterile plastic containers. During sample collection disposable sterile gloves were used in order to prevent additional microbial contamination of the material. After preliminary sanitization of external genital organs cervical swabs were taken from animals into sterile tubes with Ames transport medium. Sterile swabs and gynecological speculum were used in that procedure.

For further studies, pooled tests were used, every pool was determined by the number of samples taken from animals of all experimental groups of each dairy farm. As a result, 20 pooled milk samples and 20 pooled samples of cervical swabs were obtained.

\subsection{Conduction of microbiological studies with culture-based methods}

Bacteriologic examination included inoculation of the material on nutrient media, isolation and identification of pure cultures and detection of drug susceptibility. Cultural properties (morphological, tinctorial) were analyzed by visualizing the grown cultures on solid and liquid nutrient media. On solid media the size of the colonies, color, transparency, shape, presence of pigment, hemolysis around the colony and its character were taken into consideration. On liquid media the transparency, the presence of sediment (bottom growth) or a film on the surface of the medium were noted. The bacterial biochemical properties were defined by their enzymatic saccharolytic activity and their ability to utilize nutrients under aerobic and anaerobic cultivation conditions. After studying the morphological and cultural properties, differential tests were performed with pure cultures of microorganisms.

The following reference strains of microorganisms were used to check the nutrient media and to establish control in order to confirm the growth properties of the nutrient media: Candia albicans NCTC 885-653, Enterococcus faecalis ATCC 19433/ NCTC 775, Aspergillus brasiliensis ATCC 9642, Staphylococcus aureus ATCC 25923, Escherichia coli ATCC 25922, Pseudomonas aeruginosa ATCC 9027, Salmonella abony GISK 103/39, Bacillus subtilis ATCC 6633.

In the research accumulation media and differential diagnostic nutrient media were used: GMF-broth (NICF LLC, Russia), medium No. 10 (FBUN SSC PMB, Russia), cetrimide agar (Pronadisa Conda, Spain), culture medium for the detection of streptococci (NICF LLC, Russia), Endo media (FBUN GNTs PMB, Russia), VSA medium (FBUN GNTs PMB, Russia), Hektoen enteric agar (FBUN GNTs PMB, Russia), Czapek's medium (Biocompass- 
S LLC, Russia), Levin medium (FBUN GNTs PMB, Russia), Ploskirev medium (FBUN SSC PMB, Russia), medium No.2 (FBUN SSC PMB, Russia), Hektoen enteric agar (Pronadisa Conda, Spain), Columbia agar, with additives (Pronadisa Conda, Spain), XLD agar (FBUN SSC PMB, Russia), Olkelnitsky medium, Simmons medium, Ressel media (Russia), Kligler medium.

The biochemical properties of bacterial cultures were determined according to the instructions for the reagent kits. Biochemical assay plates were used in the research for differentiation of enterobacteria (PBDE), as well as a set of reagents for identification and differentiation of interspecies microorganisms of the genus Salmonella "DS-DIFSALMONELLA", gram-negative non-fermenting bacteria "DS-DIF-NEFERMI", microorganisms of the genus Staphylococus (I -STAFI-16 "Scientific and Production Association" Diagnostic Systems "LLC, Russia). The results of biochemical reactions were interpreted by using visual accounting, which allows you to identify different bacteria that are included in Bergey's manual (1997). The enzymatic and biochemical properties of bacteria were assessed by tests including the detection of urease, $\beta$-D-galactosidase, $\beta$ glucosidase, phosphatase, lysine decarboxylase, ornithine decarboxylase, arginine dihydrolase, nitrite reductase, formation of hydrogen sulfide, indole, acetoin; acetylmethylcarbinol, fermentation of glucose, sucrose, mannitol, trehalose, lactose, mannose, xylose, ribose, cellobiose, malonate, citrate, sodium citrate with glucose, inositol, sorbitol, arabinose, maltose.

The pathogenicity of the isolated microorganisms was defined on laboratory white mice. For the inoculation freshly isolated 18-24-hour cultures of bacteria were exploited. The grown culture was diluted with saline in ratio 1:10, 1:100, 1:1000 and injected 0,5 ml intraperitoneally in each mouse. For each dilution no less than 4 animals were picked out. The observation of animal's condition carried on for 7 days. In case of absence of animal's mortality inside the experimental groups, the isolated cultures of microorganisms were considered non-pathogenic.

The plasma coagulation test was performed using standardized, lyophilized rabbit plasma (NPO Microgen JSC, Moscow, Russia). To set up the reaction, 18-24-hour isolated culture was added into $0,5 \mathrm{ml}$ of plasma (diluted in a ratio of 1:5), afterwards the tubes were placed in a thermostat with the temperature regime range $(37.5 \pm 0.5){ }^{\circ} \mathrm{C}$, the presence of plasma clotting was checked after 1, 2, 5, 18, 24 hours. Pathogenic microorganisms produce coagulase enzyme, which coagulates the plasma. The reaction is considered positive regardless of the plasma coagulation time and clot viscosity.

The hemolytic properties of bacteria were determined using Petri dishes with Columbia agar with the addition of 5\% sterile defibrated sheep blood, incubated at a temperature of $(37.5 \pm 0.5){ }^{\circ} \mathrm{C}$ for 48 hours. In the presence of an $\alpha$ or $\beta$ hemolysis zone around the grown colony, the reaction was considered positive, in the absence of a hemolysis zone - negative.

\subsection{Statistical data processing}

Statistical data processing was conducted with standardized analysis package by Microsoft Excel 2010. In order to demonstrate effect size, data were expressed as a percentage of control values.

\subsection{The results of the research}

Following studies involving the use of cultural methods had shown that compound of cow's reproductive tract microbiota consists of opportunistic and commensal microflora, including 
such microorganisms and microfungus as: S.aureus, S.lugdunensis, Streptococcus spp., Enterobacter spp., Enterococcus faecium, Enterococcus faecalis, Escherichia coli, Proteus vulgaris, Bacillus spp., Mucor spp., Penicillum spp., Aspergillus spp (Table 1).

S. aureus in quantity of 14 isolates was obtained from $70 \%$ of milk samples and 10 isolates from $50 \%$ of cervical swabs samples. 4 cultures of $S$. lugdunensis were recovered in $5 \%$ of milk samples and $15 \%$ of cervical swabs samples. However, it ought to be acknowledged that in our earlier studies this particular microorganism was not detected and firstly appeared in current research. S.lugdunensis is typical not only for human and goat normal flora, but it is also a zoonotic pathogen responsible for community-acquired and nosocomial infections in immunocompetent and immunosuppressed patients, and had also been identified as an etiological agent that can cause abortion in dairy cattle $[16,17]$. Nevertheless, in veterinarian medicine pathogenic potential of S.lugdunensis is still understudied and requires further research investigation.

Cultures of Streptococcus spp. were identified in 14 milk samples and 19 cervical swabs samples (70\% and 95\%, consequently). Enterobacter spp. was obtained from significantly fewer samples $-10 \%$ of milk and cervical swabs samples.

In investigated clinical material microorganisms from the genus enterococci were identified. Enterococcus faecium in quantity of 8 isolates was recovered from milk samples and 13 isolates from cervical swabs samples (40\% and 60\%, consequently). Enterococcus faecalis was isolated from $30 \%$ of milk samples (6 isolates) and $35 \%$ of cervical swabs samples (7 isolates). Bacteria of the genus Enterococcus belong to the group of potentially pathogenic microorganisms, which are known to be an etiological factor of infectious inflammatory diseases in animals. The pathogenicity is associated with surface proteins involved in the processes of adhesion and invasion, cytolysin and aggression enzymes (protease, gelatinase, hyaluronidase) [18].

Isolates of E.coli were obtained from $50 \%$ of milk samples (10 isolates) and $80 \%$ of cervical swabs samples (16 isolates). Identification of Bacillus spp. in $30 \%$ of milk samples and $50 \%$ of cervical swabs samples was an indication of suitable environmental condition (e.g. in animal's bedding) for colonization of spore-forming bacteria. Into the mammary gland Bacillus spp. most often enter intramammary, for example, when drugs are administered without proper sanitization of the teat. 1 isolate of Proteus vulgaris was recovered in cervical swabs of cows, as well as 1 isolate of Pseudomonas aeroginosae.

Table 1. Analysis of microbiota composition in cows of experimental groups on the basis of microbiological studies by cultural methods, the number of isolates

\begin{tabular}{|c|c|c|c|c|c|c|c|c|}
\hline \multirow[b]{2}{*}{$\begin{array}{c}\text { Species of } \\
\text { microorganisms }(n=153)\end{array}$} & \multicolumn{2}{|c|}{ Group $1 *$} & \multicolumn{2}{|c|}{ Group $2 * *$} & \multicolumn{2}{|c|}{ Group $3 * * *$} & \multicolumn{2}{|c|}{$\begin{array}{l}\text { Group } \\
4 * * * *\end{array}$} \\
\hline & 兰 & 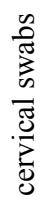 & 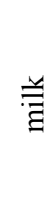 & $\begin{array}{l}\frac{\infty}{0} \\
\frac{\pi}{3} \\
\frac{\pi}{0} \\
\sum_{0}^{0} \\
0\end{array}$ & 关 & 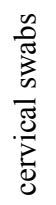 & 㲖 & 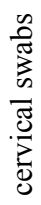 \\
\hline S.aureus $(n=24)$ & 2 & 4 & 4 & 2 & 3 & 2 & 5 & 2 \\
\hline S.lugdunensis $(n=4)$ & - & 1 & - & - & 1 & 1 & - & 1 \\
\hline Streptococcus spp. $(n=33)$ & 1 & 5 & 5 & 5 & 4 & 5 & 4 & 4 \\
\hline Enterobacter spp. $(\mathrm{n}=4)$ & - & 2 & 2 & - & - & - & - & - \\
\hline $\begin{array}{l}\text { Enterococcus } \\
\text { faecium }(\mathrm{n}=21)\end{array}$ & - & 2 & 3 & 4 & 2 & 4 & 3 & 3 \\
\hline
\end{tabular}




\begin{tabular}{|l|c|c|c|c|c|c|c|c|}
\hline $\begin{array}{l}\text { Enterococcus faecalis } \\
(\mathrm{n}=13)\end{array}$ & 2 & 4 & - & 1 & 2 & 1 & 2 & 1 \\
\hline Escherichia coli (n=26) & 2 & 4 & 3 & 3 & 3 & 4 & 2 & 5 \\
\hline Proteus vulgaris (n=1) & - & - & - & 1 & - & - & - & - \\
\hline $\begin{array}{l}\text { Pseudomonas aeroginosae } \\
\text { (n=1) }\end{array}$ & - & - & - & - & - & - & - & 1 \\
\hline Mucor spp. (n=6) & - & 1 & - & 4 & - & - & 1 & - \\
\hline Penicillum spp. (n=2) & - & - & - & - & - & 1 & - & 1 \\
\hline Aspergillus spp. (n=2) & - & 1 & - & 1 & - & - & - & - \\
\hline Bacillus spp. (n=16) & 2 & 2 & 1 & 2 & 2 & 3 & 1 & 3 \\
\hline
\end{tabular}

* Group 1 - animals with no signs of pathology;

** Group 2 - animals with inflammatory process in the mammary gland;

*** Group 3 - animals with inflammatory process in the reproductive system;

$* * * *$ Group 4 - animals with inflammatory process in the mammary gland and the reproductive system.

The dominant microscopic molds isolated from clinical specimens were Mucor spp. (5 isolates), Penicillum spp. was recovered in fewer quantities (2 isolates). In samples of cervical swabs, micromyces of the genus Aspergillus were also obtained.

Therefore, a total of 143 isolates of pathogenic and opportunistic microorganisms and 10 isolates of microscopic molds were recovered from biological material. Analysis of the distribution of identified isolates between experimental groups of animals showed that the proportion of isolated microorganisms is approximately the same in each group. Most of the isolates were identified in groups of cattle with the signs of inflammatory diseases of the reproductive tract (endometritis, vaginitis) and cows with synchronous inflammatory lesions of reproduction organs and the mammary gland $-25,9 \%$ in each group from the total number of isolates. The least number of isolates was obtained from group of cattle with no clinical manifestation of reproductive organs lesions or diseases of the mammary gland $-23,0 \%$ from the total number of isolates (Table 1). In the group of clinically healthy control subjects the largest number of isolates was obtained from cervical swabs $(72.7 \%)$, in the group of cows with mastitis, isolates from the milk samples and cervical swabs were recovered in equal proportions. In the third and the fourth group the largest number of isolates was obtained from the cervical swabs $(54,0 \%)$.

In case of inflammatory process development in the mammary gland and reproductive organs of cattle, the discovered microbiota was approximately identical (Figure 1). Streptococcus spp. (24,5\%), St.aureus (22,6\%), Enterococcus faecium (15,1\%), E.coli $(15,1 \%)$ were dominant group in the spectrum of microorganisms isolated from milk samples, which allows to estimate the role of that microflora in the development of the pathological process.

In the structure of isolates that were obtained from the cervical swabs from the group of cows with inflammatory pathology, the majority of microorganisms consisted of Streptococcus spp. (21,5\%), E.coli (18,5\%), Enterococcus faecium (16,9\%), Bacillus spp. $(12,4 \%)$. The distinctive aspect of present study was shedding of Enterobacter spp. only from milk samples $(3,8 \%)$, as well as identification of P.vulgaris and P.aeruginosae only in reproductive tract swabs (1,5\% and 1,5\%, consequently). The major part of microscopic mold fungi isolates was obtained from cervical swabs (Mucor spp. $-6,2 \%$, Penicillum spp. $-3,1 \%$, Aspergillus spp. - 1,5\%), in milk samples only Mucor spp. $(1,9 \%)$ was detected. 

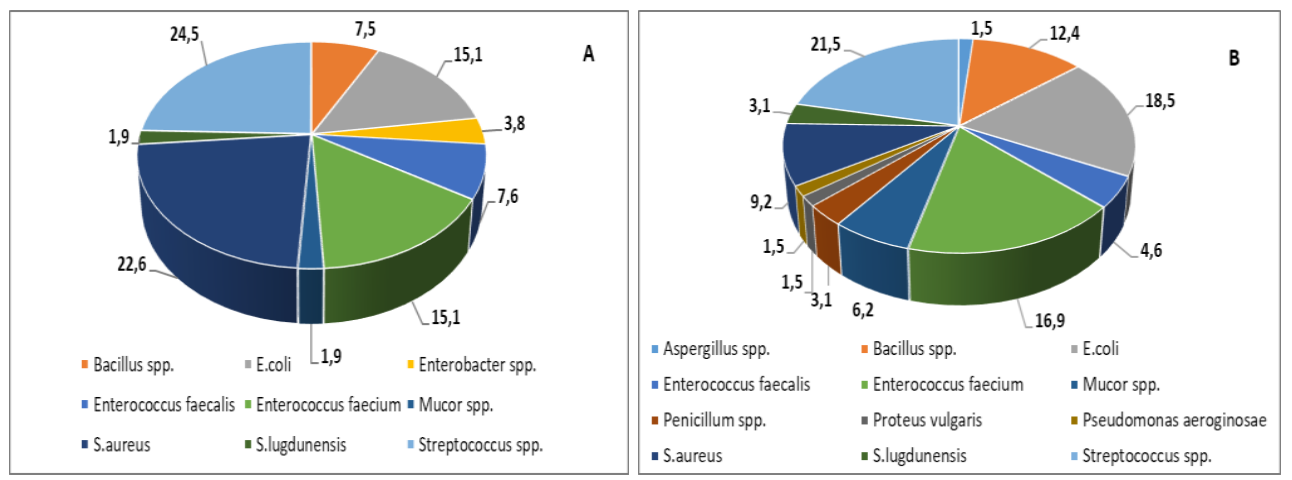

Fig. 1. The structure of the mammary gland microbiota $(\mathrm{A})$ and the reproductive tract microbiota (B) in the group of cows with inflammatory process, $\%$.

In the group of clinically healthy cows the spectrum of microorganisms that was obtained from milk samples was significantly narrow, the main part of microflora's structure consisted of S.aureus, Enterococcus faecalis, E.coli, Bacillus spp. - in equal amounts about 22,2\%. It is characteristic that no microscopic fungi molds were found in clinical material from healthy cows. The larger number of isolates was obtained from cervical swabs than from milk samples, the main part of microorganisms composed of Streptococcus spp. (19,2\%), S.aureus, Enterococcus faecalis, E.coli - in equal proportions about 15,4\%. Among fungi molds only 2 isolates were recovered from genus of Mucor and Aspergillus (Figure 2).

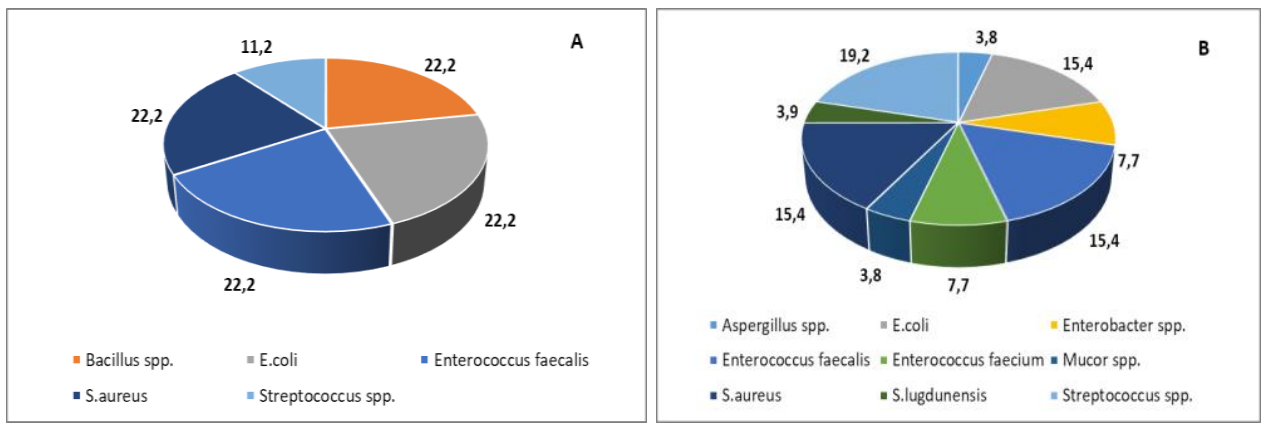

Fig. 2. The structure of the mammary gland microbiota and the reproductive tract microbiota in cows with no signs of pathology, $\%$.

In addition to studying the phenotypic characteristics of the isolated microorganisms, the evaluation of biochemical properties of isolates such as S.aureus, S. lugdunensis, E.coli, Pseudomonas aeruginosa was conducted. $20,8 \%$ of S.aureus isolates were identified, those microorganisms apart from standard biochemical properties fermented cellobiose as well (16.7\% - the part of isolates from milk samples). About $16,7 \%$ of S.aureus isolates from the mammary gland secretion lacked phosphatase. In comparison to the standard biochemical profile, $50 \%$ of $S$. lugdunensis isolates fermented lactose, 1 of isolates simultaneously lacked $\beta$-glucosidase and urease and the presence of arginine dihydrolase was determined. Enzymes like urease and arginine dihydrolase can be attributed to the pathogen factors of microorganisms, the latter destroys the amino acid arginine, which is necessary for the normal functioning of the human and animal body.

All isolates of S.aureus and S.lugdunensis had shown a negative reaction in the formation of acetylmethylcarbinol (acetoin), indicating a change in the mechanisms of the VogesProskauer reaction, which presumably means phenotypic manifestation of new genovariants 
of the studied strains of microorganisms. Similar changes, for instance, have been studied in V.cholarae strains [18].

All isolates of E.coli were compliant with standardized biochemical profile of microorganisms according to the conducted tests.

A distinctive feature of the detected Pseudomonas aeruginosa isolate was a positive reaction to the utilization of inositol, hydrolysis of esculin and a negative reaction to the presence of phosphatase and intra-reductase.

The results indicated from the research show the variation of phenotypic properties of some isolates of microorganisms that were obtained from milk and cervical samples of cows. That variation includes the appearance of biochemical properties that can determine the pathogenicity of bacteria.

Evaluation of virulence factor testing and characteristics of pathogenicity (hemolytic efficiency, coagulase) in obtained isolates showed that all isolates of S.aureus caused clotting of rabbit plasma after 18-24 hours, in other words, they were coagulase-positive. Isolates of S.lugdunensis were coagulase-negative. Other microbiological cultures were examined on Columbia agar supplemented with 5\% defibrinated sheep blood. In all cases, after 48 hours, the zonal changes in agar media, such as clear zone surrounding the bacterial growth was not indicated, which means the absence of hemolytic properties in the tested microorganisms. The pathogenicity testing of isolated microorganisms conducted on laboratory white mice that were inoculated intraperitoneally, showed no mortality of animals registered within 7 days.

\section{Results and Discussion}

Thus, all detected microorganisms isolated from the reproductive tract and the mammary gland secretions of cows with inflammatory lesion and without pathology belong to opportunistic microorganisms. Their participation in the development of the pathological process can be attributed to their quantity (microbial load), long-term persistence in the macroorganism, the relationship between associates, the state of immunobiological resistance of the macroorganism and the ability of microorganisms to influence on various factors of the host's immunity. Moreover, in the research work of Russian scientists, the ability of enterococci to inactivate such factors of innate immunity as lysozyme and carnosime was discovered, and the values of persistent characteristics turned out to be higher in clinical isolates [19]. From this perspective, it is more significant to evaluate the disease-inciting factors of the conditional pathogen, and especially the pathogenicity causing level of its activity. The individual changes in the biochemical profile of some isolates identified by us may indicate their acquirement of new phenotypic properties due to the genetic variability of microorganisms, which may also be indicative of the enhancement of some virulence factors.

\section{Conclusion}

1. As a result of conducted microbiological studies 143 isolates of pathogenic and opportunistic microorganisms were obtained from the biological material. The discovered microbiota of the mammary gland and reproductive organs with inflammatory process development in cattle was approximately identical.

2. The main part of the mammary microbiota spectrum accrue to Streptococcus spp. $(24,5 \%)$, St.aureus (22,6\%), Enterococcus faecium $(15,1 \%)$, E.coli $(15,1 \%)$, which allows us to highlight the crucial role of this microflora in the pathological process development. The majority of isolates that were obtained from cervical swabs in cows with inflammatory 
lesions, consisted of Streptococcus spp. (21,5\%), E.coli (18,5\%), Enterococcus faecium (16,9\%), Bacillus spp. (12,4\%).

3. The phenotypic characteristics of the isolated microorganisms are given, including the assessment of the biochemical properties of the S.aureus isolate. Apart from standardized biochemical properties $20,8 \%$ of S.aureus isolates had an ability to ferment cellobiose and $16,7 \%$ of S.aureus isolates from milk samples lacked enzyme phosphatase. $50 \%$ of S.lugdunensis isolates fermented lactose, 1 isolate simultaneously lacked $\beta$-glucosidase and urease, the presence of arginine dihydrolase was detected. All isolates of S.aureus and S.lugdunensis had shown a negative reaction to the formation of acetylmethylcarbinol (acetion). The specific feature of identified Pseudomonas aeruginosa isolates was a positive reaction on the inositol utilization, esculin hydrolysis and a negative reaction on the presence of phosphatase and nitrate reductase. The assessment of virulent and pathogenic properties of microorganisms had evaluated that all isolates of S.aureus were coagulase-positive.

\section{References}

1. T. Andrews, D.A. Neher, T.R. Weicht, J.W. Barlow, PLoS One, 14(11), e0225001 (2019)

2. Irina Donnik, Olga Bykova, Anna Krivonogova, International transaction journal of engineering management \& Applied sciences \& Technologies, 10(2), 203-209 (2019)

3. C.J. Doyle, D. Gleeson, P.W. O'Toole, P.D. Cotter, Appl Environ Microbiol, 83(2), e02694-16 (2016)

4. P. Rainard, Vet Res., 48(1), 25 (2017) 10.1186/s13567-017-0429-2.

5. M.C. Montel, S. Buchin, A. Mallet, C. Delbes-Paus, D.A. Vuitton, N. Desmasures, et al. Int J Food Microbiol, 177, 136-154 (2014) 10.1016/j.ijfoodmicro.

6. M.F. Addis, A. Tanca, S. Uzzau, G. Oikonomou, R.C. Bicalho, P. Moroni, Mol BioSyst,12(8), 2359-2372 (2016) 10.1039/c6mb00217.

7. M.V. Ryaposova, I.A. Shkuratova, D.M. Kadochnikov, M.N. Tarasenko, The Russian journal "Problems of Veterinary Sanitation, Hygiene and Ecology", 3 (15), 53-55 (2015)

8. K.N. Galvão, R.C. Bicalho, S.J. Jeon., Dairy Sci., 102(12), 11786-11797 (2019) doi: 10.3168/jds.2019-17106. Epub 2019 Oct 3.

9. S.J. Jeon, K.N. Galvão, Genomics Inform, 16(4), e21 (2018) doi: 10.5808/GI.2018.16.4.e21.

10. L.R. Knudsen, C.C. Karstrup, H.G. Pedersen, J.S. Agerholm, T.K. Jensen, K. Klitgaard, Vet Microbiol., 175(2-4), 319-24 (2015) doi: 10.1016/j.vetmic.2014.12.006.

11. S.G. Moore, A.C. Ericsson, S.K. Behura, W.R. Lamberson, T.J. Evans, M.S. McCabe, S.E. Poock, M.C. Lucy, BMC Genomics, 20(1), 405 (2019) doi: 10.1186/s12864-0195797-8.

12. Y. Wang, J. Wang, H. Li, K. Fu, B. Pang, Y. Yang, Y. Liu, W. Tian, R. Cao. Theriogenology,108, 306-313 (2018) doi: 10.1016/j.theriogenology.2017.12.028

13. D.E. Gomez, K.N. Galvão, J.C. Rodriguez-Lecompte, M.C. Costa, Vet Clin North Am Food Anim Pract, 35(3), 485-505 (2019) doi: 10.1016/j.cvfa.2019.08.002. Review.

14. M.C. Espeche, M. Pellegrino, I. Frola, A. Larriestra, C. Bogni, M.E.F. Nader-Macías, Anaerobe, 18(1), 103-109 (2012) 10.1016/j.anaerobe.2012.01.002. 
15. I.M. Donnik, I.A. Shkuratova, A.G. Isayeva, N.A. Vereshyak, A.S. Krivonogova, J.B. Beykin, V.S. Portnov, M.I. Barashkin, O.G. Loretz, Agrarian Bulletin of the Urals, 1 (93), 26-28 (2012)

16. P. Ardigò, M. D'Incau, S. Pongolini, J Vet Diagn Invest, 26(6), 818-820 (2014) doi:10.1177/1040638714550182

17. Ardigò Paolo, Mario D'Incau, Stefano Pongolini, Journal of Veterinary Diagnostic Investigation, 26(6), $\quad 818-820 \quad$ (2014) DOI: $\quad 10.1177 / 1040638714550182$ jvdi.sagepub.com

18. S.P. Zadnova, A.A. Kritsky, N.A. Plekhanov, N.P. Guseva, Yu.V. Lozovsky, N.I. Smirnova, Problems of Particularly Dangerous Infections, 2, 62-68 (2019) doi.org/10.21055/0370-1069-2019-2-62-68

19. E.E. Kochkina, T.M. Pashkova, T.M. Sychova, Bulletin of the Byryat State Academy of Agroculture, 9, 70-75 (2017) 